IRSTI 29.27.07

https://doi.org/10.26577/phst.2021.v8.i2.05

\title{
Influence of an external electric field on plasma parameters around an isolated dust particle
}

\author{
A. Fedoseev1,** iD, M. Salnikove ID and G. Sukhinin ${ }^{2}$ \\ ${ }^{1}$ Joint Institute for High Temperatures RAS, Izhorskaya st. 13 Bd.2, 125412, Moscow, Russia \\ ${ }^{2}$ Institute of Thermophysics SB RAS, 1, Lavrentyev Ave., 630090, Novosibirsk, Russia \\ "e-mail: alex.fed245@gmail.com
}

\begin{abstract}
The paper presents new numerical results on the behavior of plasma parameters around an isolated charged dust particle under the action of the external electric field. For the first time, the model takes into account the dependence of mean electron energy on the reduced electric field strength. As a result of the calculations, the dependencies of self-consistent spatial distributions of the electron and ion densities and electric potential around the dust particle on reduced external electric field strength were obtained. These distributions were analyzed through the expansion into Legendre polynomials. The processes of ion focusing and wake formation behind the dust particle were studied. The dust particle charge and the dipole moment of the "ion cloud - dust particle" are calculated for different values of the reduced electric field and ion mean free paths. It is shown that in the determination of electron density spatial profile and the dust particle charge the dependence of electron temperature on electric field strength plays a significant role.
\end{abstract}

Key words: dusty plasma, dust particle charging, dipole moment, wake, plasma polarization.

PACS number(s): 52.27.Lw, 52.35.Tc, 52.20.-j

\section{Introduction}

Dusty or complex plasma is a new field of plasma physics studding the interaction of solid micron-sized particles immersed into typical lowtemperature plasma (see e.g. recent reviews $[1,2]$ ). Many different phenomena associated with the dusty plasma have been studied experimentally and numerically: dust grains charging [3], the formation of crystalline and liquid structures [4,5], phase transitions between these structures $[5,6]$, dustacoustic waves [7], the orientation of non-spherical dust particles in a discharge [8], and many others.

It is well known that in streaming plasma or in an external electric field the pseudo-periodic oscillating structures in electric potential and ion density arise behind a dust particle [18,19,20]. These phenomena are responsible for the dust particles ordering. Such a periodic structure is called a wake, and is a consequence of ion focusing behind the dust grain. The most popular approaches for studying wakes are PIC [9-11] and LR [12,13] numerical methods.

In previous works [14-19], the behavior of plasma parameters around an isolated charged dust particle under the action of the external electric field was studied. As a result of the calculations, the dependencies of self-consistent spatial distributions of the electron and ion densities and electric potential around the dust particle on reduced external electric field strength were obtained. The processes of ion focusing and wake formation behind the dust particle were studied. The dust particle charge and the dipole moment of the "ion cloud - dust particle" are calculated for different values of dust particles size, electric field strength and ion mean free paths. However, in all these studies the parameter $\tau=T_{e} / T_{i}$, i.e. the ratio of electron and ion temperatures, was the constant parameter (typically $\tau=100$ ), which did not depend on the external electric filed. It is well known that the electron mean energy is a function of the reduced electric field specific for the type of a buffer gas. In present work, the dependence of mean electron energy on the reduced electric field was taken into account for the first time.

\section{Model}

A detailed description of the numerical model used for the calculation of the plasma parameters around an isolated dust particle was presented 
elsewhere [14-19]. Let us just remind the following points. The geometry of the computational domain was taken as a parallelepiped. An impenetrable sphere of radius $r_{0}<<\lambda_{i}$ placed in the center of this domain plays the role of the solid dust particle, where $\lambda_{i}$ is the ion Debye length.

To start the calculations, an ion is generated with the random coordinates and velocities. The speed of ions is taken according to the Maxwell distribution. Newton's motion equations were used to calculate the trajectory of this generated ion. In these equations, the action of electric field induced by the charged dust particle and external electrostatic field on the ion trajectory is taken into account. During its movement the ion could either fall on a dust particle or fly away the computational domain or collide with a neutral atom.

The modeling domain was subdivided into cells $(i, j)$ according to cylindrical coordinates $\rho$ and $z$, respectively. The volume of each cell is determined by:

$$
V_{i, j}=2 \pi \rho_{i} \Delta \rho_{i} \Delta z_{j}
$$

During ion traveling through the cell $(i, j)$, the time $T_{i, j}$, which the ion spent in this cell, is accumulated. Then, this time is normalized to the volume of the cell $V_{i, j}$. The obtained value is summed up with the already accumulated time statistics for this cell:

$$
n_{i}(i, j)=n_{i}(i, j)+\frac{T_{i, j}}{V_{i, j}} .
$$

We use the dimensionless parameters in the calculations, i.e. the dimensionless charge of the dust particle $Q^{\prime}$ and the dimensionless external electric field $E^{\prime}$ given by the expressions:

$$
Q^{\prime}=\frac{e^{2} Z_{d}}{\lambda_{i} k T_{i}}, E^{\prime}=\frac{e E \lambda_{i}}{k T_{i}}
$$

At the beginning of calculations, the following spatial distribution of the electric potential was used:

$$
\begin{gathered}
U_{0}(\rho, z)=-\frac{Q^{\prime}}{r} \exp (-r)- \\
-E^{\prime} z, \quad r=\sqrt{\rho^{2}+z^{2}}
\end{gathered}
$$

From the statistics obtained for every cell, the dimensionless distribution of space charge $n(\rho, z)$, is calculated:

$$
n(\rho, z)=\frac{n_{i}(\rho, z)-n_{e}(\rho, z)}{n_{\infty}},
$$

were $n_{\infty}$ is the plasma density far from the dust particle. The spatial distribution of electron density $n_{e}$ is assumed to be equal to the Boltzmann distribution $n_{e}(\rho, z)=n_{\infty} \exp \left(U(\rho, z) /\left(k T_{e}\right)\right)$.

The self-consistent spatial distribution of electric potential in the system is then calculated as:

$$
\begin{gathered}
U(\rho, z)=-\frac{Q^{\prime}}{r}+ \\
+\iiint_{V_{s s s t}} \frac{n\left(\rho^{\prime}, \phi^{\prime}, z^{\prime}\right) \rho^{\prime} d \rho^{\prime} d \phi^{\prime} d z^{\prime}}{\left|\vec{r}-\vec{r}^{\prime}\right|}
\end{gathered}
$$

The general iterative scheme for calculation of a steady state self-consistent solution for all plasma parameters is as follows:

1) The calculation of ion trajectories under the action of the spatial distribution of electric potential (6). After the ion collides with the neutral atom or fall onto the dust particle surface, a new ion is simulated. The statistics is accumulated for all ions.

2) The distribution of the space charge (5) is determined and the self-consistent electric potential of the system is calculated (6). The charge of the dust particle is calculated from the condition that the flows of ions and electrons to its surface are equal to each other.

3) The iterative procedure is repeated until the full convergence of all spatial distributions, including the charge of the dust particle.

For small values of the external electric field, the spatial distributions of electron density $n_{e}(r, \theta)$, ion density $n_{i}(r, \theta)$ and electric potential $U(r, \theta)$ weakly deviate from spherical symmetric $(r$ and $\theta$ are spherical coordinates). In this case, $U(r, \theta)$ can be determined through the expansion of the space charge distribution $n(r, \theta)$ into the Legendre polynomials (spherical harmonics):

$$
\begin{gathered}
n(r, \theta)=n_{i}(r, \theta)-n_{e}(r, \theta)= \\
=\sum_{k=0} n_{k}(r) P_{k}(\cos \theta),
\end{gathered}
$$

where 


$$
n_{k}(r)=\frac{2 k+1}{2} \int_{0}^{\pi} n(r, \theta) P_{k}(\cos \theta) \sin \theta d \theta .
$$

It should be noted that the zero isotropic term of the expansion, $n_{0}(r)$, determines the plasma charge around the dust particle:

$$
Q_{p l}=\frac{1}{2} \int_{0}^{\infty} \int_{0}^{\pi} n(r, \theta) r^{2} \sin \theta d \theta d r=\int_{0}^{\infty} n_{0}(r) r^{2} d r
$$

and the first isotropic term of the expansion, $n_{l}(r)$, determines the dipole moment of a "dust particle ion cloud" system:

$$
\begin{gathered}
P_{p l}=\frac{1}{2} \int_{0}^{\infty} \int_{0}^{\pi} n(r, \theta) r^{3} \cos \theta \sin \theta d \theta d r= \\
=\frac{1}{3} \int_{0}^{\infty} n_{1}(r) r^{3} d r .
\end{gathered}
$$

\section{Results}

In calculations, a spherical dust particle of radius $r_{0}=1 \mu \mathrm{m}$ is considered. Ion temperature is assumed to be equal to the room temperature, i.e. $k T_{i}=0.03 \mathrm{eV}, T_{i}=T_{g}$. The ion Debye length is set to be $\lambda_{i}=10^{-2} \mathrm{~cm}$, i.e. $r_{0}=10^{-2} \lambda_{i}$. Ion mean free path for the resonant charge exchange collisions with neutrals (argon) is taken in the interval $l_{i}=$ 2.5-10 $\lambda_{i}$. The interval of external electric field values is $E^{\prime}=0-0.6$, i.e. $E=0-1.5 \mathrm{~V} / \mathrm{cm}$. The gas (argon) density $n_{0}=1 /\left(2^{1 / 2} \sigma l_{i}\right)$ is determined by the ion mean free path $l_{i}$ and the resonant charge exchange collision cross section $\sigma$ (for argon [20]).

In the previous authors works as well as in other literature, the parameter $\tau=T_{e} / T_{i}$ was the given parameter, where $T_{e}$ is the electron temperature. In this paper, we take into account that electron temperature is the function of the reduced external electric field $E / N$. We use the dependence of the electron mean energy $<\varepsilon>=$ $3 / 2 k T_{e}$ versus reduced electric field $E / N$ calculated with the help of BOLSIG+ solver [21] (see Figure 1) to obtain the dependence $T_{e}(E / N)$. This dependence plays significant role in the determination of electron density spatial profile and of electron flux towards the dust particles, i.e. influences the dust particle charge.

Figure 2 presents the dependence of the particle charge on electric field strength both in dimensionless and dimension forms. The aim of the calculations is to determine the role of the ion mean free path $l_{i}$ on the dust particle charge at the same electron temperature. It is seen that different curves for different ion mean free paths substantially differ from each other $(40 \%)$. The dust particle charge and charge number increase almost linearly with the electric field strength excepting the region of low electric filed, i.e. the low electron temperature. It should be noted that at constant parameter $\tau$ considered in previous papers the charges differ more slightly.

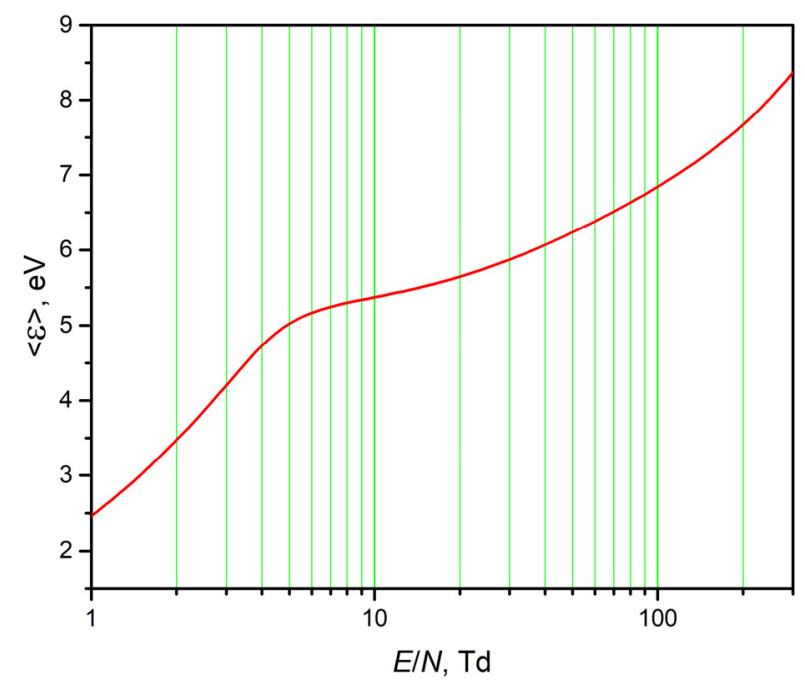

Figure 1 - Electron mean energy $<\varepsilon>$ versus reduced electric field $E / N[21]$ 

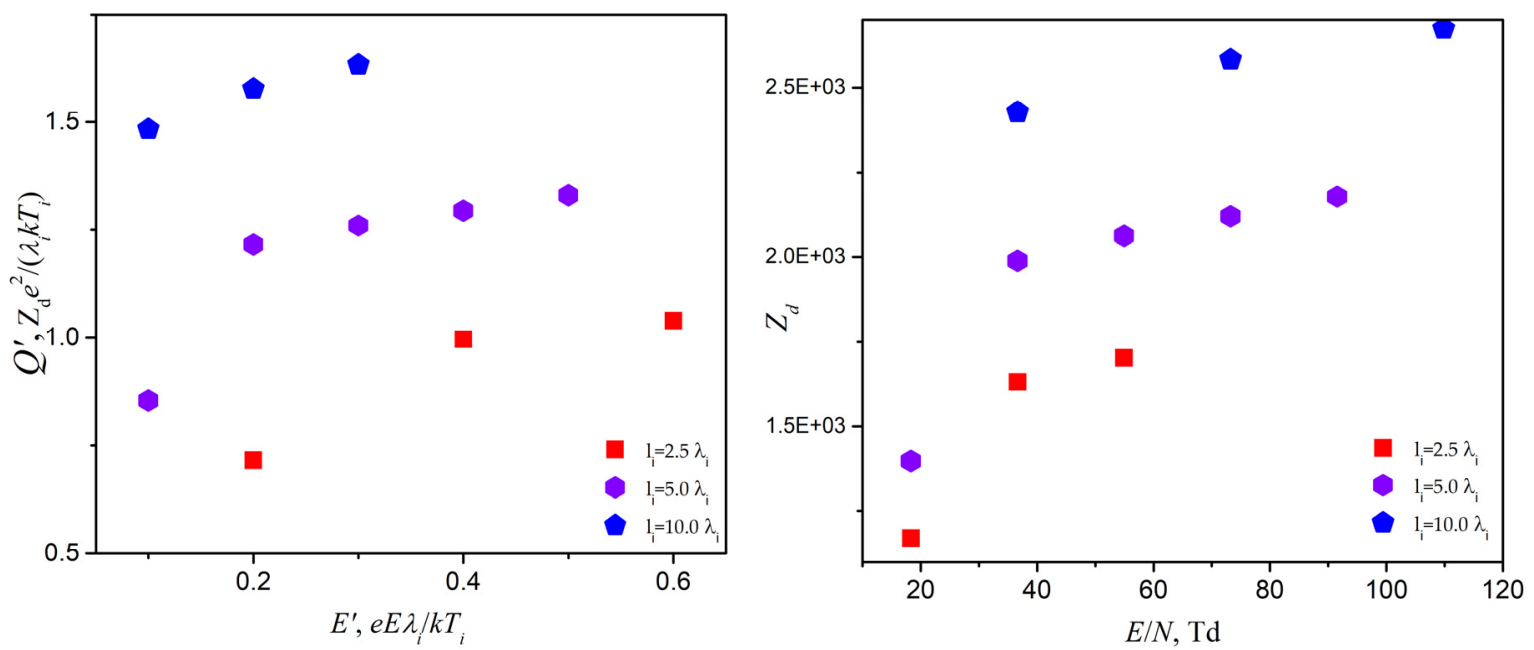

Figure 2 - Dimensionless dust particle charge $Q^{\prime}$ versus dimensionless electric field $E^{\prime}$ (left figure) and dust particle charge number $Z_{d}$ versus reduced electric field $E / N$ (right figure) for different values of ion mean free paths
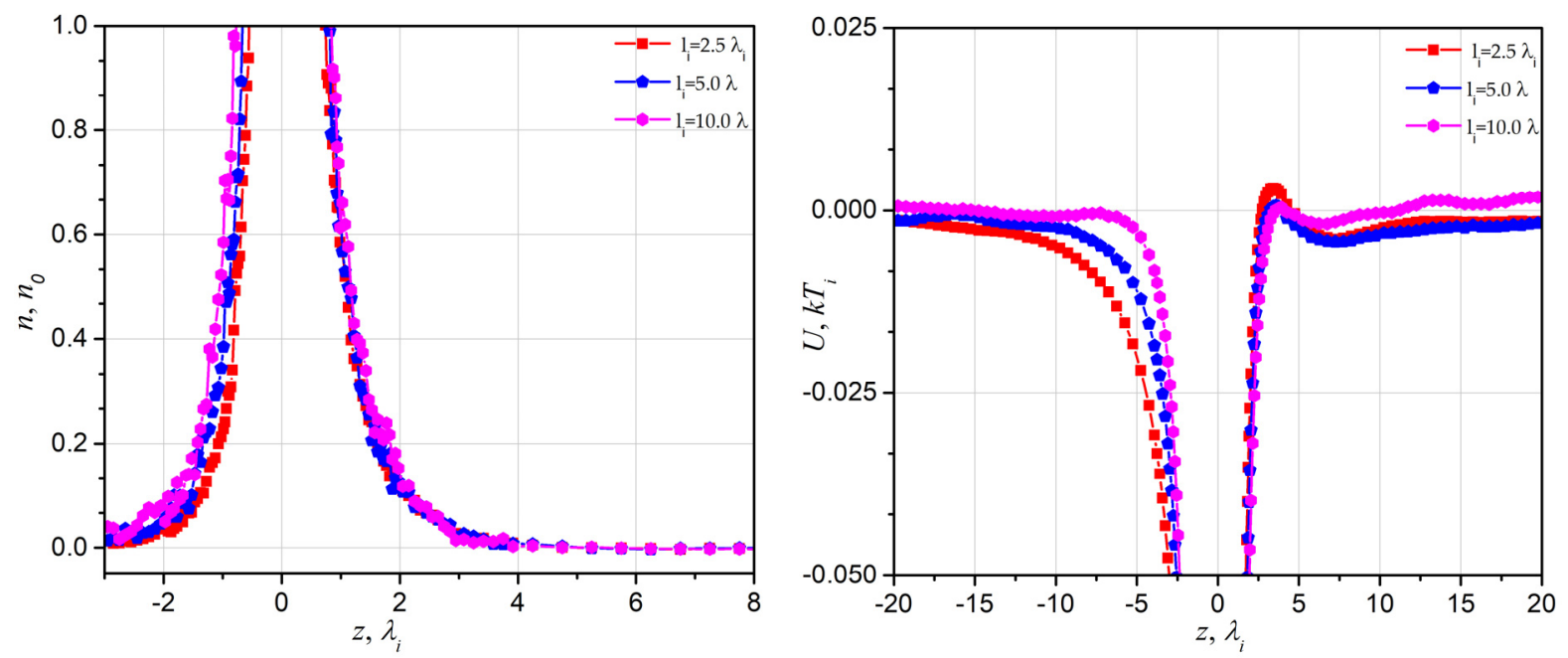

Figure 3 - The sections of the spatial distributions of the space charge density $n(z, \rho=0)$ (left figure) and the electric potential $U(z, \rho=0)$ (right figure) for different values of ion mean free paths. Reduced electric field $E / N=37 \mathrm{Td}$

Figure 3 shows sections $(\rho=0)$ of the longitudinal distributions of the space charge density $n(z, \rho=0)$ and the electric potential $U(z, \rho=0)$. The value of the reduced electric field strength for all curves is the same $E / N=37 \mathrm{Td}$. It is interesting to note that the right parts of the space charge density and the electric potential are substantially deviate for different ion mean free paths and the right parts are almost coincide with each other. Thus, the wakes are formed almost at the same distances. The more the ion mean free path the more amplitude of the oscillations of the electric potential behind the dust particle due to the fact that the collisions of ions with neutrals destruct the wakes.

Figure 4 presents radial distributions of zero and first harmonics multiplied by special compounds, i.e. functions $n_{0}(r) r^{2} / Q^{\prime}$ and $n_{l}(r) r^{3} l_{i}^{1 / 2}$. The result for $n_{0}(r) r^{2} / Q^{\prime}$ shows that at different electron energies the shape of the charge distribution is uniform. This is very interesting taking into account that the case for $l_{i}=2.5$ has a strongly narrowed ion cloud. The result for $n_{l}(r) r^{3} l_{i}^{1 / 2}$ is also interesting and shows that the 
unscreened part (closer to the particle than $3 \lambda_{i}$ ) behaves like a functional from the root of the $l_{i}$.

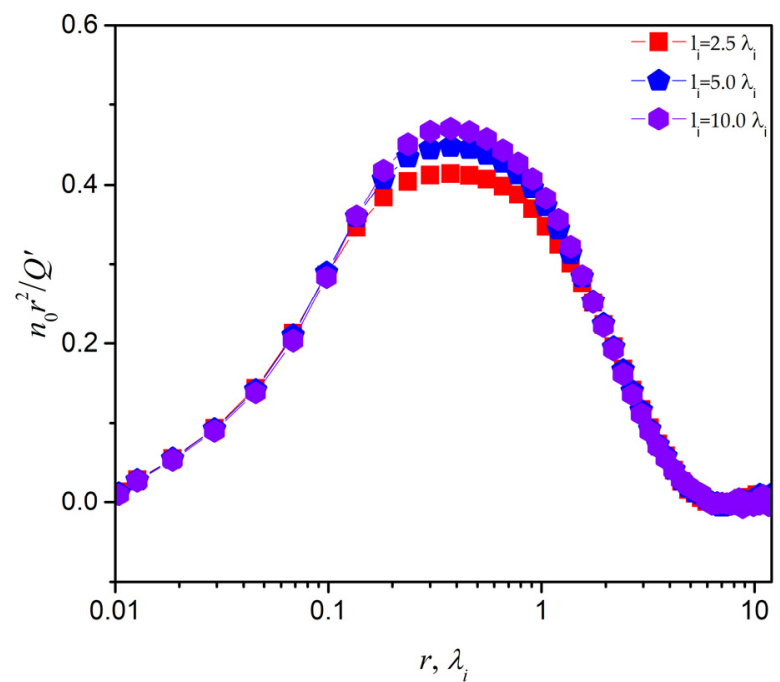

The area beneath function $n_{l}(r) r^{3}$ multiplied by the $l_{i}^{1 / 2}$ is equal for different $l_{i}$ (see Figure 5).

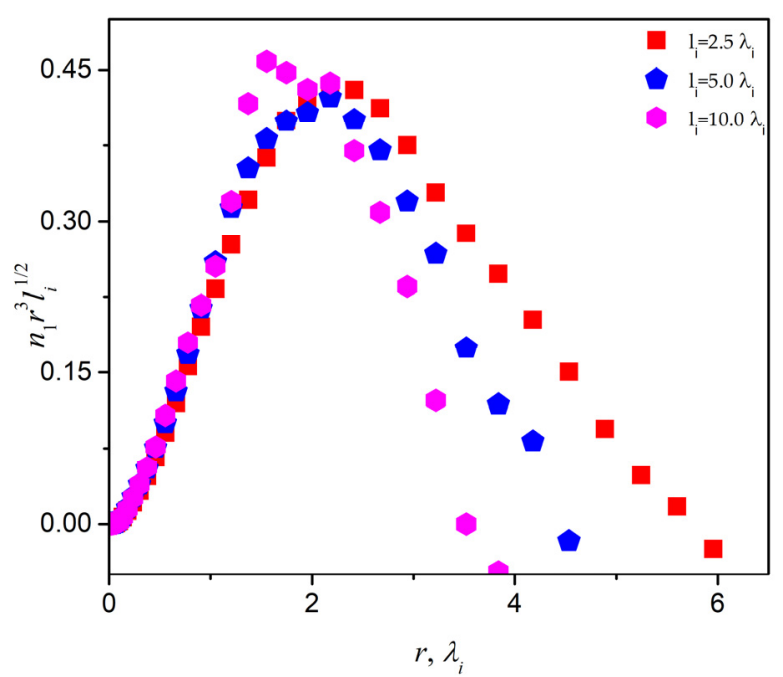

Figure 4 - Functions $n_{0}(r) r^{2} / Q^{\prime}$ (left figure) and $n_{l}(r) r^{3} l_{i}^{1 / 2}$ (right figure) for different values of ion mean free paths. Reduced electric field $E / N=37 \mathrm{Td}$
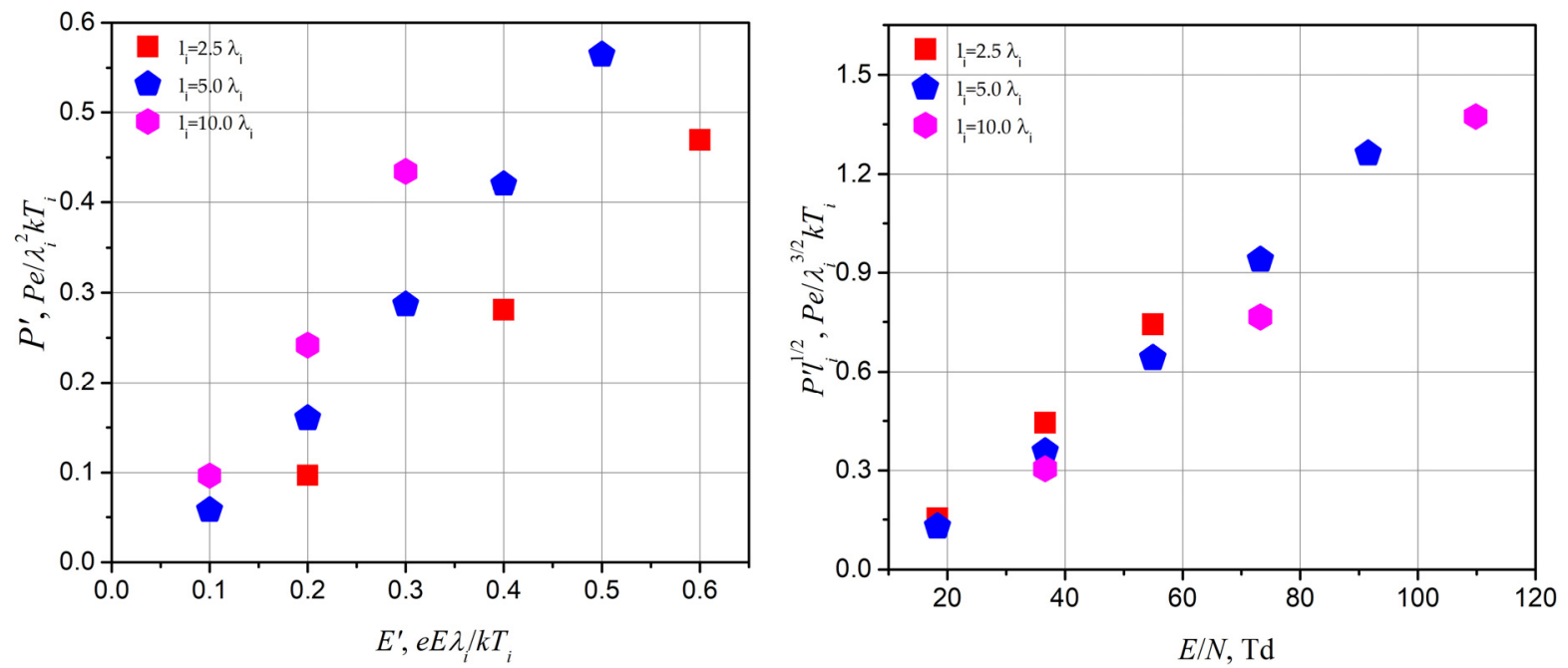

Figure 5 - Dimensionless dipole moment versus dimensionless electric field (left figure) and function $P^{\prime} l_{i}^{1 / 2}$ versus reduced electric field $E / N$ for different values of ion mean free paths

The dipole moment of the "dust particle - ion cloud" ought also be reviewed for the same electron energy for various $l_{i}$. The dipole moment $P_{p l}$ is calculated by Equation (10). Figure 5 (left figure) shows the dependence of dimensionless dipole moment $P^{\prime}$ on dimensionless electric field $E^{\prime}$ for different ion mean free paths. It is seen that the curves increase almost linearly with the electric field. The more $l_{i}$ the more value of the dipole moment due to ions could orbit around the particle more time contributing to plasma anisotropy. The dependence of the function $P^{\prime} l_{i}^{1 / 2}$ on the reduced electric filed is presented on the right image of the Figure 5. It is seen that different curves for different $l_{i}$ are almost coincide with each other.

As a result of this study, it was shown that, despite taking into account the change in the 
electron energy, and, as a consequence, the change in the dust particle charge value, the dipole moment can still be characterized as a linear function of the external electric field (as in [17]). Thus, taking into account the electron energy does not affect the dependence of the ion cloud anisotropy on the magnitude of the external field.

\section{Conclusions}

In the paper the results of the numerical modeling of the plasma parameters around an isolated charged dust particle under the action of an external electric field are presented. Previously developed computational model was expanded by taking into account the dependence of mean electron energy on the reduced electric field strength. As a result of calculations, the dependencies of self-consistent spatial distributions of the electron and ion densities, electric potential around the dust particle were obtained for different values of ion mean free path and reduced electric field. It is shown that the accounting for the dependence of electron temperature on electric field strength plays significant role in the determination of electron density spatial profile and the dust particle charge by means of electron flux towards the dust particle.

\section{References}

1 M.Y. Pustylnik, A.A. Pikalev, A.V. Zobnin, I.L. Semenov, H.M. Thomas, O.F. Petrov. (2021) Physical aspects of dust-plasma interactions // Contributions to Plasma Physics. - 2021. - P. e202100126. https://doi.org/10.1002/ctpp.202100126

2 R. Merlino. Dusty plasmas: from Saturn's rings to semiconductor processing devices // Advances in Physics: X. - 2021. - Vol. 6. - Iss. 1. - Pp. 1873859. https://doi.org/10.1080/23746149.2021.1873859

3 G. I. Sukhinin, A. V. Fedoseev. Charging of dust grains in a non-equilibrium plasma of a stratified glow

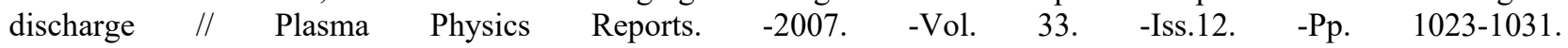
https://doi.org/10.1134/S1063780X07120070

4 H. Ikezi. Coulomb solid of small particles in plasmas // The Physics of Fluids. - 1986. - Vol. 29. - Iss. 6. Pp. 1764-1766. https://doi.org/10.1063/1.865653

5 H. M. Thomas, G. E Morfill. Melting dynamics of a plasma crystal // Nature. - 1996. - Vol. 379. - Iss. 6568. - Pp. 806-809. https://doi.org/10.1038/379806a0

6 S. A. Khrapak, B. A. Klumov, P. Huber, V. I. Molotkov, A. M. Lipaev, V. N. Naumkin, A. V. Ivlev, H. M. Thomas, M. Schwabe, G. E. Morfill, O. F. Petrov, V. E. Fortov, Y. Malentschenko, S. Volkov. Fluid-solid phase transitions in three-dimensional complex plasmas under microgravity conditions // Physical Review E. -2012. -Vol. 85. -Iss. 6. -Pp. 066407. https://doi.org/10.1103/PhysRevE.85.066407

7 P. K. Shukla, V. P. Silin. Dust ion-acoustic wave // Physica Scripta. -1992. -Vol.45. -Iss. 5. -Pp. 508-508. https://doi.org/10.1088/0031-8949/45/5/015

8 A. V. Ivlev, A. G. Khrapak, S. A. Khrapak, B. M. Annaratone, G. Morfill, K. Yoshino. Rodlike particles in gas discharge plasmas: Theoretical model // Physical Review E. -2003. -Vol.68. -Iss.2. -Pp. 026403. https://doi.org/10.1103/PhysRevE.68.026403

9 P. Ludwig, W. J. Miloch, H. Kahlert, M. Bonitz. On the wake structure in streaming complex plasmas // New J. Phys. -2012. -Vol.14. -Pp. 053016. https://doi.org/10.1088/1367-2630/14/5/053016

10 I. H. Hutchinson. Nonlinear collisionless plasma wakes of small particles // Phys. Plasmas. -2011. -Vol. 18. Pp. 032111. https://doi.org/10.1063/1.3562885

11 I. H. Hutchinson. Intergrain forces in low-Mach-number plasma wakes // Phys. Rev. E. -2012. -Vol. 85, -Pp. 066409. https://doi.org/10.1103/PhysRevE.85.066409

12 R. L. Dewar., D. Leykam. Dressed test particles, oscillation centres and pseudo-orbits // Plasma Phys. Control. Fusion. -2012. -Vol. 54. -Pp. 014002. https://doi.org/10.1088/0741-3335/54/1/014002

13 R. Kompaneets, G. E. Morfill, A. V. Ivlev. Interparticle attraction in 2D complex plasmas // Phys. Rev. Lett. -2016. -Vol. 116. -Pp. 125001. https://doi.org/10.1103/PhysRevLett.116.125001

14 G.I. Sukhinin, A.V. Fedoseev, M.V. Salnikov. Polarization of a dust particle and surrounded plasma in an

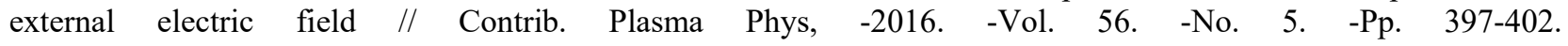
https://doi.org/10.1002/CTPP.201500128

15 G. I. Sukhinin, A. V. Fedoseev, M. V. Salnikov, A. Rostom, M. M. Vasiliev, O. F. Petrov. Plasma anisotropy around a dust particle placed in an external electric field // Phys. Rev. E. -2017. -Vol. 95. -Pp. 063207. https://doi.org/10.1103/PhysRevE.95.063207 
16 G.I. Sukhinin, A.V. Fedoseev, M.V. Salnikov, A. Rostom. Plasma polarization and wake formation behind a dust particle in an external electric field // IEEE Transactions on Plasma Science. - 2018. - Vol. 46. - No. 4. - Pp. 749-754. https://doi.org/10.1109/TPS.2017.2775656

17 G.I. Sukhinin, A.V. Fedoseev, M.V. Salnikov. Effect of ion mean free path length on plasma polarization behind a dust particle in an external electric field // Contributions to Plasma Physics. -2019. -Pp. e201800152. https://doi.org/10.1002/CTPP.201800152

18 G.I. Sukhinin, A.V. Fedoseev, M.V. Salnikov. The influence of dust particle geometry on its charge and plasma potential // Contributions to Plasma Physics. -2019. -Pp. e201800153. https://doi.org/10.1002/ctpp.201800153

19 G. Sukhinin, M. Salnikov, A. Fedoseev. Plasma anisotropy around non-spherical conductive dust particle. Physical Sciences and Technology. -2019. -Vol. 6. -No.2. -Pp. 37-43. https://doi.org/10.26577//phst-2019-2-p5

20 S.A. Maiorov. Ion drift in a gas in an external electric field // Plasma Phys. Rep. -2009. -Vol. 35. -Pp. 802812. https://doi.org/10.1134/S1063780X09090098

21 G. J. M. Hagelaar, L. C. Pitchford. Solving the Boltzmann equation to obtain electron transport coefficients and rate coefficients for fluid models // Plasma Sources Sci. Techn. -2005. -Vol.14. -Pp. $722-733$. https://doi.org/10.1088/0963-0252/14/4/011 